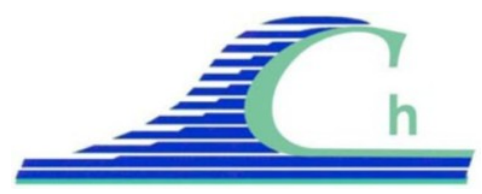

XII ${ }^{\text {̀̀mes }}$ Journées Nationales Génie Côtier - Génie Civil

Cherbourg, 12-14 juin 2012

DOI:10.5150/jngcgc.2012.031-H（C) Editions Paralia CFL

disponible en ligne - http://www.paralia.fr - available online

\title{
Stability of a stratified shear flow for the modelling of estuary bed
}

\author{
Alice HARANG ${ }^{1,2}$, Olivier THUAL ${ }^{1,2}$ \\ 1. Université de Toulouse, INPT, UPS, IMFT, Allée Camille Soula, \\ F-31400 Toulouse, France. \\ 2. CNRS, IMFT, F-31400 Toulouse, France. \\ harang@imft.fr ; thual@imft.fr
}

\begin{abstract}
:
To improve the understanding of mud resuspension and the behaviour of mud flow in estuaries, a parametric stability analysis of a shear flow is carried out with a model of two miscible fluid layers of different properties. The shear layer is modelled by asymmetric $\operatorname{erf}(z)$ vertical profiles of the same thickness for each quantities. The upper layer, composed of water, is considered as a Newtonian fluid. The lower layer mud flow is also considered as a Newtonian fluid of high density and high viscosity. A linear stability study, realized with the code LiSa developed at IMFT (Institut de Mécanique des Fluides de Toulouse), explores the influence of the principal control parameters: the Richardson number, the Reynolds number and viscosity ratio between the two fluids. Direct numerical simulations (DNS) performed with the code JADIM of IMFT are used to compute the temporal evolution of these flows and are compared with the linear stability study. Both approaches describe consistently the development of the bidimensional primary instability. The computed critical Richardson number is close to 0.25 . Results show that for high Reynolds numbers, evaluated at the interface, there is no influence of the viscosity ratio with respect to the primary instability. For Reynolds number lower than $10^{3}$, the viscosity has a destabilizing effect: a bigger difference of viscosity leads to a more important growth rate. This property can result in higher instability growth rates for highly viscous mud flows when submitted to a velocity shear in real estuaries. Secondary instabilities and the other processes leading to an eventual three-layer flow are documented through DNS simulations. Different secondary instabilities are observed depending on the Reynolds number at the interface. Likely outcomes of this study are new parameterizations of the bed sediment flux in realistic modelling of estuaries.
\end{abstract}

\section{Keywords:}

Estuary - Turbidity maximum - Mixing - Stratification - Mud flow - Resuspension Sedimentary transport - Linear stability 


\section{Introduction}

Estuaries are very complex systems, and one of the sticking point is fluid mud. Its behaviour is still misunderstood, as pointed out MC ANALLY (2007) who says: "the fondamental dynamics of the processes and the formulation of constitutive relations are dependent on empirical relationships based on small data sets". Through the observations of the Amazon estuary, KINEKE et al. (1996) stresses the importance of mud flow on sedimentary process and hydrodynamic processes as it reduces the boundary shear stress. DOXARAN et al. (2009), using observations based on satellites, shows relations between the sediment discharge in the ocean at the Gironde estuary and the presence of mud flow in the lower estuary. Laboratory experiences have also been realized to provide a better understanding of mudflow process. GRATIOT et al. (2005) and SANCHEZ \& LEVACHER (2008) respectively demonstrates the influence of isotropic turbulence on settling velocity and on the entrainment of plastic muds. Using numerical simulations, WINTERWERP (2002) presents the relation between flocculation and settling velocity. Concerning the entrainment, KRANENBURG \& WINTERWERP (1997) propose a model based on the kinetic energy balance. Their study focuses on the entrainment of mudflow at the lutocline. This last interface can be considered as a mixing layer of two different miscible fluids. Stability and mixing of a shear stratified flow is studied by CORCOS \& SHERMAN (1984) while its transition to mixing layer is documented by CORCOS \& LIN (1984) and CAULFIELD \& PELTIER (2000). Shear flow with viscous variation is explored by YIH (1967). The present work aims to improve models of entrainment proposed by PARTHENIADES (1963), PARCHURE \& METHA (1985) and ODD \& COOPER (1989), which are used in many numerical models such as TELEMAC_3D or SIAM-3D for example. Present study fills out HARANG et al (2010) by studing more precisly the effect of control parameters. The goal is to compute more accurately the sediment flux of estuaries and thus deal with pollution or coastal settlement.

\section{A conceptual model of mud flow}

\subsection{Mixing layer profiles}

The water-mud flow interface is modeled by a two layer flow with continuous interface. Vertical profiles are based on the erf function near the interface, in an empirical way, to qualitatively model a simple shear. To model vertical profiles, we consider the group of functions $F(\lambda, Z)$ defined by:

$$
F(\lambda, Z)=\left\{\begin{array}{c}
\lambda\left[1+\operatorname{erf}\left(\frac{Z}{\lambda}\right)\right] \text { if } Z \leq 0, \\
1-(1-\lambda)\left[1-\operatorname{erf}\left(\frac{Z}{1-\lambda}\right)\right] \text { if } Z \geq 0,
\end{array}\right.
$$




\section{XII ${ }^{\text {èmes }}$ Journées Nationales Génie Côtier - Génie Civil \\ Cherbourg, 12-14 juin 2012}

where $Z$ is the non-dimensional vertical coordinate to be specified later, the origin of which is located at the interface. The parameter $\lambda$ controls the asymmetry of the fields located on both sides of the inflexion point, with continuity of the profile and its derivative around this point.

We consider the vertical profiles of the longitudinal velocity $u(z)$, of the density $\rho(z)$ and of the dynamic viscosity $\mu(z)$ given by the relations:

$$
\begin{aligned}
& u(z)=u_{2} F\left(\lambda, \frac{z-h}{\delta_{u}}\right), \\
& \rho(z)=\left(\rho_{2}-\rho_{1}\right) F\left(\lambda, \frac{z-h}{\delta_{\rho}}\right)+\rho_{1}, \mu(z)=\left(\mu_{2}-\mu_{1}\right) F\left(\lambda, \frac{z-h}{\delta_{\mu}}\right)+\mu_{1},
\end{aligned}
$$

where $\delta_{u}, \delta_{\rho}$ and $\delta_{\mu}$ are the thicknesses respectively associated to the profiles $u(z), \rho(z)$ et $\mu(z)$. Here, we restrict ourselves to the case where these three thicknesses are equal to $\delta=0.3 \mathrm{~m}$. The boundaries of the simulation field are defined by the equations $\mathrm{z}=0$ and $z=3 h$, the height $z=h=1 \mathrm{~m}$ being the interface location. In addition, we have chosen $\lambda=0.1$, since diffusion is assumed to be greater in water than in mud flow. The corresponding profiles are presented in figure 1 .

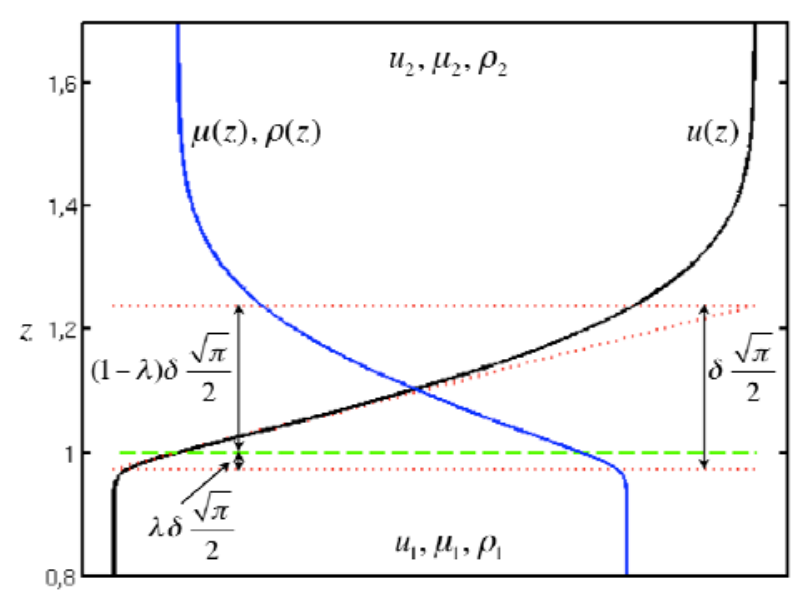

Figure 1. Initial profiles of $u(\mathrm{z}), \rho(\mathrm{z})$ and $\mu(\mathrm{z})$ on the part $\mathrm{z} \in[0.8 \mathrm{~m}, 1.7 \mathrm{~m}]$ of the domain $\mathrm{z} \in[0,3 \mathrm{~h}]$, with $h=1 \mathrm{~m}, \delta=0.3 \mathrm{~m}$ and $\lambda=0.1$.

The choice of a 2D configuration has been justified by an exploration of 3D configurations, in which we have observed the development of instabilities in the flow direction. However the Squire Theorem, valid for homogeneous flows, cannot exclude instabilities with a finite spanwise wave number. 


\subsection{Control parameters}

In our configuration, the mixing of the two fluids is concidered: the upper layer is of density $\rho_{2}$ and dynamic viscosity $\mu_{2}$ while the lower layer is of density $\rho_{1}$ and dynamic viscosity $\mu_{1}$. The Atwood number $A t=\left(\rho_{1^{-}} \rho_{2}\right) /\left(\rho_{1}+\rho_{2}\right)$ is fixed to 0.15 . The three other non-dimensional numbers, relevant for our study, are defined by:

$$
\operatorname{Re}=\frac{u_{h} \delta}{\mu_{h} / \rho_{h}}, R i=\frac{g}{\rho_{h}} \frac{\left(\rho_{2}-\rho_{1}\right) \delta \sqrt{\pi}}{2 u_{2}^{2}} e t W=\log _{10}\left(\frac{\mu_{1}}{\mu_{2}}\right) \text {, }
$$

where $\rho_{h}=\rho(h)=\left(\rho_{2}-\rho_{1}\right) \lambda+\rho_{1}, \mu_{h}=\mu(h)=\left(\mu_{2}-\mu_{1}\right) \lambda+\mu_{1}$ and $u_{h}=u(h)=u_{2} \lambda$ are respectively the density, the viscosity and the velocity at the interface. The Reynolds number $R e$ and the gradient Richardson number $R i$ are evaluated at the interface $z=h$ defined by the inflexion of initial profiles, and $W$ is the logarithm of the viscosity ratio between mud flow and water.

\subsection{Model equations and method of resolution}

Our model of mixed layer is studied through the following system of equations:

$$
\begin{aligned}
& \nabla \cdot \underline{u}=0, \frac{d \underline{u}}{d t}=-\frac{1}{\rho} \nabla P+\underline{g}+\frac{1}{\rho} \nabla\left[\mu\left(\nabla \underline{u}+{ }^{t} \nabla \underline{u}\right)\right], \\
& \frac{\partial \varphi}{\partial t}+\nabla \cdot(\varphi \underline{u})=0, \\
& \rho=\rho_{2} \varphi+\rho_{1}(1-\varphi), \mu=\mu_{2} \varphi+\mu_{1}(1-\varphi),
\end{aligned}
$$

which links incompressible Navier-Stokes equations (4.a), where $g$ is the acceleration of gravity, to the transport equation (4.b) of a volume fraction $\phi$ which controls, through relations (4.c), the kinematic mixing of fluid particles of non-homogeneous density $\rho$ and dynamic viscosity $\mu$. Molecular diffusion of sediments is neglected here (see DEARDORFF \& WILLIS, 1982). Both entities are then mixed and followed through the volume fraction of lower layer fluid (mud flow) in the fluid, $\phi$. So, in these simulations, density and viscosity are linear functions of the volume fraction and then vary in the same way.

These equations are solved by the Navier-Stokes solver, JADIM, developed at IMFT (Institut de Mécanique des Fluides de Toulouse), specialized in multiphase incompressible flows. Based on primitive variables (velocity, pressure), the equations are solved by a finite volumes method. The code is $2^{\text {nd }}$ order accurate in space and time, thanks to a $3^{\text {rd }}$ order Runge-Kutta temporal scheme and a semi-implicit Crank-Nicolson scheme for diffusive terms. The equations are discretized using a $2^{\text {nd }}$ order centered scheme with staggered variables (CALMET \& MAGNAUDET 1996; LEGENDRE \& MAGNAUDET 1998). The transport equation of the presence rate is discretized using the WENO scheme. The version used in the present study solves the system of 


\section{XII ${ }^{\text {èmes }}$ Journées Nationales Génie Côtier - Génie Civil \\ Cherbourg, 12-14 juin 2012}

equations (4) and has been validated for a viscosity gradient by ERN et al. (2003) and for a density gradient by HALLEZ (2007).

The stability of this interface is also studied through the consideration of little perturbations of a base flow. The linear stability code LiSa (ANTKOWIAK \& BRANCHER, 2004; 2007) is used for this purpose, which provides a global vision of the problem. This code computes the eigen modes of the system of equations (4) linerarized around the base flow (2). Given a parallel base flow, a perturbation (defined by its wave vector) and specific numerical parameters, LiSa provides the eigen values and their associated modes, using a spectral method based on Chebyshev polynomials. So, for each wave vector, one can extract the most unstable mode and its growth rate. Two versions are used. The first is classical: a real mapping is employed to go from the physical grid point space ot the spectral space of Chebyshev coefficients. The second version, following the idea of FABRE et al. (2006), uses a complex mapping between the physical and spectral spaces which allows us to find more converged eigen values in some configurations.

\section{Results}

\subsection{Linear stability analysis}

In this first part, series of linear stability computations have been realised to study the influence of control parameters presented above. In these computations, the configuration parameters $(\lambda, \delta, h, H)$ are constant as well as the density of each layer. The velocity of the upper layer fixes the Richardson number, $R i$ (the lower layer being steady). Viscosities of the two layers allow us to choose the Reynolds number $R e$ at the interface and the viscosity ratio, $W$. The growth rate of the more unstable mode is computed for the normalized wavenumber of the perturbation $k^{*}$ and is presented in figure 2 for $R i=0.15, W=0$. We choose to scale the wave number with half the thickness of the interface : $k^{*}=k \delta \sqrt{\pi} / 4$; the phase velocity by $u_{2} / 2$ and the growth rate is normalized by $\sigma^{*}=\sigma \delta \sqrt{\pi} /\left(2 u_{2}\right)$.

We first observe, in figure 2, the variation of the growth rate $\sigma^{*}$ as function of the wave number $k^{*}$. The part of the spectrum that is represented with continuous lines is obtained with a distribution of spatial grid points obtained with a classical mapping. The part represented with dashed lines has been computed with a complex mapping, in which the coordinates of the grid points are replaced by imaginary numbers. This method has been developed to get rid of the critical layer singularities and thus compute more eigenvalues near the marginal stability curve, especially for large wave number. The configuration is unstable for a Richardson number under a critical Richardson number $R i_{c} \sim 0.25$. Contrary to the classical study of tanh mixing layer (see HAZEL, 1972), higher instable wave number are observed because of the asymmetry of vertical profiles. 
The growth rate and the critical Richardson number are a little higher when $\mathrm{Re}$ is decreased and W increased. This is further illustrated in figure 4, which shows isocontours of the growth rates 0.02 and 0.08 in the wave number and Reynolds number plane for different viscosity ratios $W$. This figure shows that, for Reynolds number higher than $10^{3}$, the growth rate no longer depends on the viscosity ratio nor the Reynolds number. For Reynolds numbers smaller than this value, we observe more important growth rates and thus a larger interval of instable wave numbers: the viscosity has a destabilising influence. We can also note that a higher viscosity ratio between fluids emphasises this destabilising effect. Figure 5, which represents the evolution of the higher growth rate with the Reynolds number, presents the same influence of the viscosity ratio. It also suggests a smaller critical Reynolds for the development of the instability for important viscosity gradients.

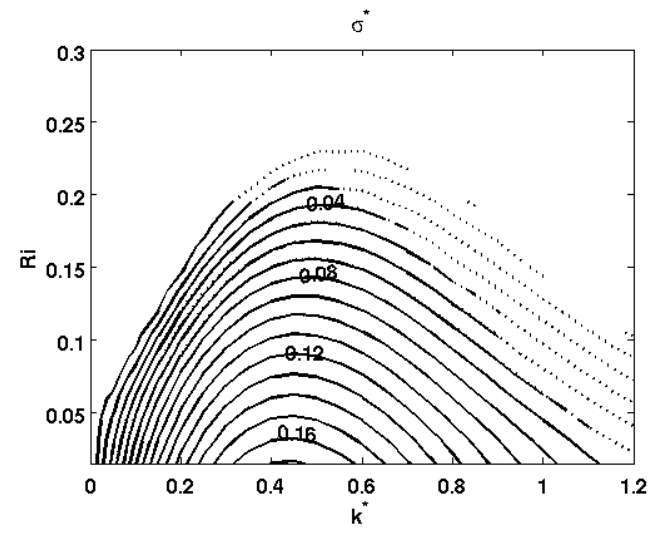

Figure 2. Growth rate $\sigma^{*}$ for $k^{*}=[0,1.2]$, $R e=96, W=3$ (... : complex mapping)

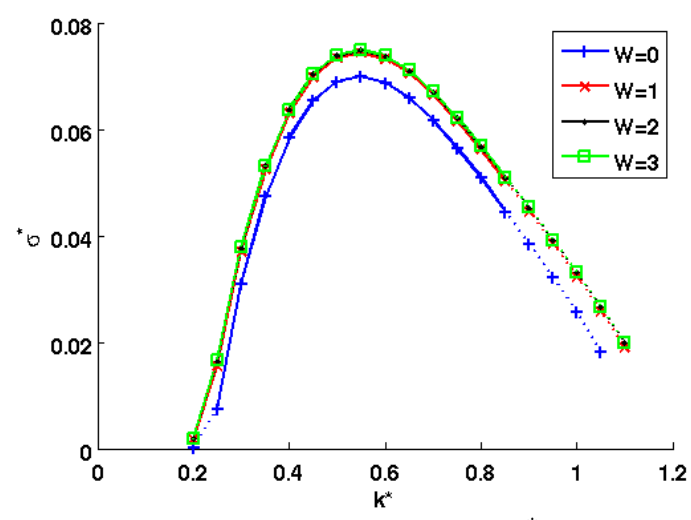

Figure 3. Growth rate $\sigma^{*}$ for $k^{*}=[0,1.2]$, $R e=100, R i=0,15$ (... : complex mapping).

\subsection{Direct numerical simulations}

Direct numerical simulations of this configuration have been realized with the code JADIM. The 2D simulations are performed on a periodic field in the streamwise direction, of $3 \mathrm{~m}$ height and $2 \pi / \mathrm{k}$ length with a resolution of $0.5 \times 0.5 \mathrm{~cm}$, and with no slip boundary condition at the bottom and free-slip condition at the top. At initial time, the parallel flow is perturbed by inserting, at low amplitude, the perturbations profiles for all quantities fields, obtained with the linear stability code LiSa.

The growth rate of instabilities is computed as the director coefficient of $0.5 \log \left(E_{k}\right)$ with the time (see figure 6), where $E_{k}$ is the kinetic energy.

A linear growth is observed for $\mathrm{Re}=10^{3}$ et $\mathrm{Re}=10^{4}$. Compared to the LiSa computation, an error of $10 \%$ on the growth rate is observed for $\operatorname{Re}=10^{3}$ and of only $3 \%$ for $\operatorname{Re}=10^{4}$. In the case of Reynolds numbers smallers than $\mathrm{Re}=10^{2}$, viscosity leads to important diffusivity of the velocity profiles. Characteristic numbers of the developpement of the 


\section{XII ${ }^{\text {èmes }}$ Journées Nationales Génie Côtier - Génie Civil \\ Cherbourg, 12-14 juin 2012}

instability and diffusion by viscosity are of the same order and we can no longer make the hypothesis of quasi-steady state for the base flow. In complement to the LiSa code, direct numerical simulations show the saturation of the instability and the development of secondary shear instabilities (see figure 7), pairing and eventually leading to a stable three layer flow. These simulations need higher resolution, secondary instability in figure 7 has been obtained with a grid of $720 \times 1200$ points (squares of $0.25 \mathrm{~cm}$ ).

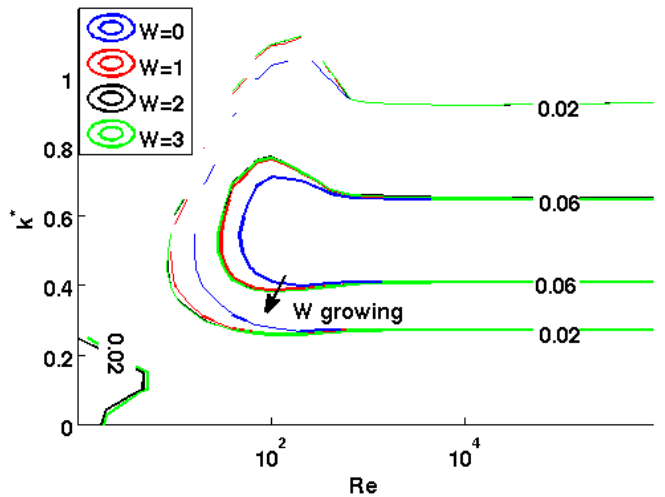

Figure 4. Iso-contours of the growth rate as function of the wave number $k^{*}$ and the Reynolds number $R e$, for $R i=0,15$, and different $W$.

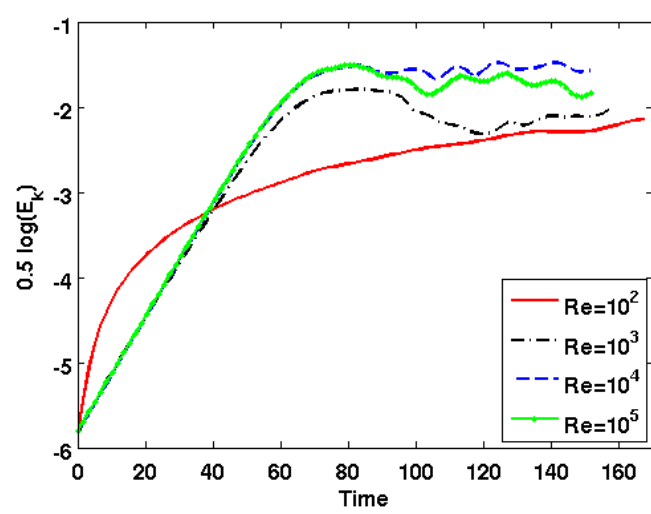

Figure 6. Representation of the kinetic energy logarithm as function of the time for different Re numbers $(\mathrm{Ri}=0.15$,

$$
\left.k^{*}=0,46, W=0\right) \text {. }
$$

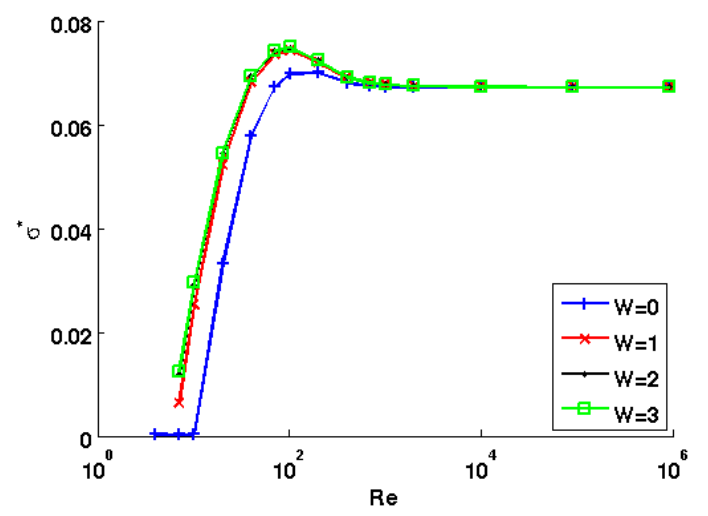

Figure 5. Maximum of the growth rate as function of the Reynolds number for different $W$.

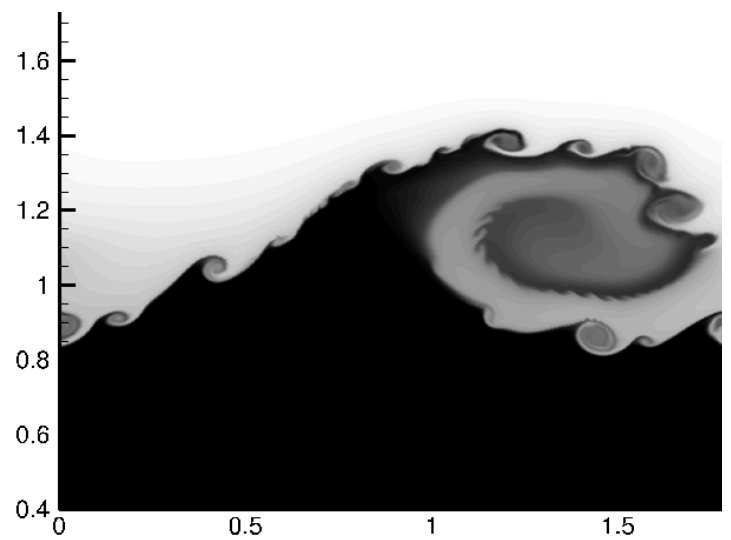

Figure 7. Representation of the density field at time $t=14$ s for $W=2, R e=10^{3}$, $k=0,46, R i=0,15$.

\subsection{Application to a scenario of mud consolidation}

If we now project our study in the natural environment, such as for the case of the mud flow, we can consider that the upper layer is water with a fixed viscosity of $\mu_{2}=10^{-3} \mathrm{~Pa}$.s, 
a density of $\rho_{2}=1000 \mathrm{~kg} \mathrm{~m}^{-3}$ and the mud flow, of density $\rho_{1}=1360 \mathrm{~kg} \mathrm{~m}^{-3}$ (MEHTA et al., 1989) and viscosity $\mu_{1}$. For a given Richardson number, if the viscosity of the mud flow is growing, the Reynolds at the interface will decrease like $10^{\mathrm{W}}$, the viscosity ratio. As presented in figure 8, for important viscosity ratios, the growth rate is more important because of both the smaller Reynolds number leading to the destabiling effect of viscosity and the viscosity ratio amplificating this effect.

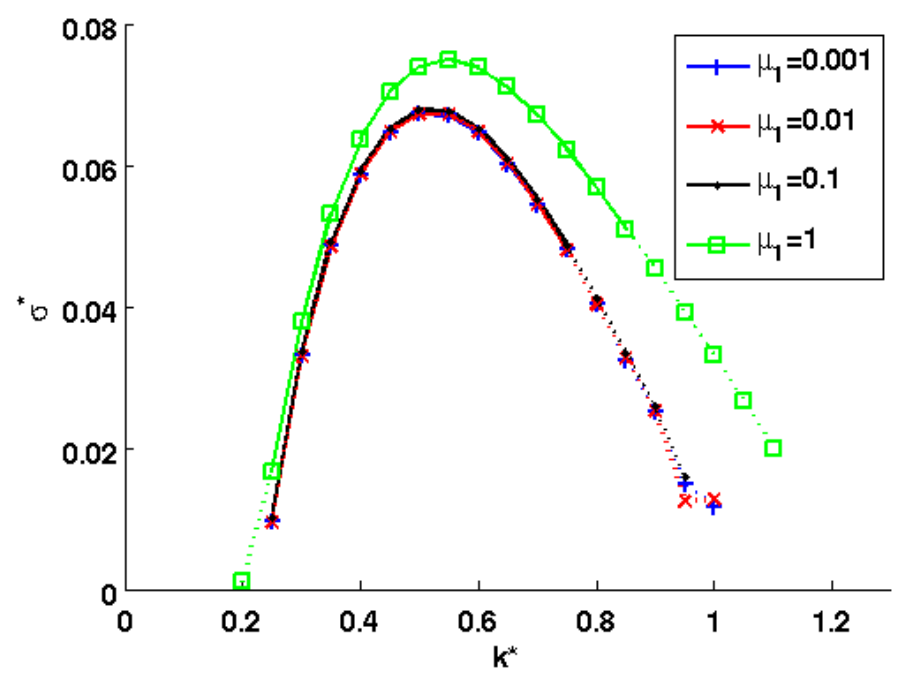

Figure 8. Instability growth rate for muds of different viscosity at mudflow - water interface, $R i=0,15$.

\section{Conclusion}

We have modeled the interface between water and mud flow by two Newtonian miscible fluids presenting strong variations of density and viscosity. The linear stability analysis explores the range of control parameter and allows a better understanding of their influence on the flow. The Richardson number of the interface, balancing stratification and shear effect, controls the heart of the Kelvin-Helmholtz instability: a Richardson behind the critical Richardson number $\left(\mathrm{Ri}_{\mathrm{c}} \sim 1 / 4\right)$ leads to instability. For large Reynolds number, there is no influence of the viscosity ratio, or the Reynolds number on the instability. Under about $10^{3}$, the Reynolds number at the interface has an influence on the instability: the viscosity has a destabilizing influence. The viscosity ratio increases this destabilizing effect. The direct numerical simulations permit a validation of these results and show their limits: for smaller Reynolds number, the hypothesis of quasi-steady state of the mean flow is not possible. Eventually, at the mud flow - water interface, if the mud flow is about 100 times more viscous than water, the growth rate will be more important because of both the influence on the Reynolds number amplified by the viscosity ratio. In the future, a comparison with visco-plastic flows is planed to have better understanding of the role of the yield stress. 


\section{XII ${ }^{\text {èmes }}$ Journées Nationales Génie Côtier - Génie Civil \\ Cherbourg, 12-14 juin 2012}

\section{Acknowledgements}
DGA
The first author is supported by a scholarship intended for $\mathrm{PhD}$ students funded by the DGA (Direction Générale de l'Armement).
This work has benefited from CICT and GENCI computational means.

\section{Bibliography}

ANTKOWIAK A., BRANCHER P. (2004). Transient energy growth for the LambOseen vortex. Physics of Fluids, Vol. 16(1), pp. L1-L4. doi:10.1063/1.1626123

ANTKOWIAK A., BRANCHER P. (2007). On vortex rings around vortices: an optimal mechanism. Journal of Fluids Mechanics, Vol. 578, pp. 295-304. doi:10.1017/S0022112007005198

CALMET I., MAGNAUDET J. (1996). Large Eddy Simulation of high-Schmidt number mass transfer in a turbulent channel flow. Physics of Fluids, Vol. 9(2), pp 438-455. doi:10.1063/1.869138

CAULFIELD C.P., PELTIER W.R. (2000). The anatomy of the mixing transition in homogeneous and stratified free shear layers. Journal of Fluid Mechanics, $\mathrm{n}^{\circ} 413$, pp 1-47. [URL http://journals.cambridge.org/action/displayAbstract?aid=12877]

CORCOS G.M., LIN S.J. (1984). The mixing layer: deterministic models of a turbulent flow. Part 2. The origin of the three-dimensional motion. Journal of Fluid Mechanics, Vol. 139, pp 67-95. doi:10.1017/\$0022112084000264

CORCOS G.M., SHERMAN F.S. (1984). The mixing layer: deterministic models of a turbulent flow. Part 1. Introduction and the two-dimensional flow. Journal of Fluid Mechanics, Vol. 139, pp 29-65. doi:10.1017/S0022112084000252

DEARDORFF J.W., WILLIS G.E. (1982). Dependence of mixed-layer entrainment on shear stress and velocity jump. Journal of Fluid Mechanics, $\mathrm{n}^{\circ}$ 115, pp 123-150. doi:10.1017/S0022112082000688

DOXARAN D., FROIDEFOND J-M., CASTAING P., BABIN M. (2009). Dynamics of the turbidity maximum zone in a macrotidal estuary (the Gironde, France): Observations from field and MODIS satellite data. Estuarine, Coastal and Shelf Science, Vol. 81, n³, pp 321-332. doi:10.1017/S0022112084000252

ERN P., CHARRU F., LUCHINI P. (2003). Stability analysis of a shear flow with strongly stratified viscosity. Journal of Fluid Mechanics, $\mathrm{n}^{\circ}$ 496, pp 295-312. doi:10.1017/S0022112003006372

FABRE D., SIPP D., JACQUIN L. (2006). Kelvin waves and the singular modes of the Lamb-Oseen vortex. Journal of Fluid Mechanics, Vol. 551, pp 235-274. doi:10.1017/S0022112005008463

GRATIOT N., MICHALLET H., MORY M. (2005). On the determination of the settling flux of cohesive sediments in a turbulent fluid. J. Geophys. Res., vol 110, ${ }^{\circ} \mathrm{C} 6$, pp C06004. doi:10.1029/2004JC002732 
HALLEZ Y. (2007). Mélange gravitationnel de fluides en géométrie confinée. PhD Report, Institut National Polytechnique de Toulouse, 140 p.

[URL http://oa.imft.fr/1851/1/hallez2007.pdf]

HARANG A., THUAL O., BRANCHER P., BONOMETTI T. (2010). Stabilité d'un écoulement cisaillé modélisant la crème de vase. Revue Paralia, Vol. 3, pp 8.1-8.12. doi:10.5150/revue-paralia.2010.008

HAZEL P. (1972). Numerical studies of the stability of inviscid stratified shear flows. Journal of Fluid Mechanics, Vol.51, n²1, pp 39-61. doi:10.1017/S0022112072001065

KINEKE G.C., STERNBERG R.W., TROWBRIDGE J.H., GEYER W. R. (1996). Fluid-mud processes on the Amazon continental shelf. Continental Shelf Research, Vol. 16, n5-6, pp 667-696. doi:10.1016/0278-4343(95)00050-X

KRANENBURG C., WINTERWERP J.C. (1997). Erosion of fluid mud layers. I: Entrainment model. Journal of Hydraulic Engineering, Vol. 123, $\mathrm{n}^{\circ}$ 6, pp 504-511. doi:10.1061/(ASCE)0733-9429(1997)123:6(504)

LEGENDRE D., MAGNAUDET J. (1998). The lift force on a spherical bubble in a viscous linear shear flow. Journal of Fluid Mechanics, Vol. 368, pp 81-126. doi:10.1017/S0022112098001621

MC ANALLY W.H. et al. (2007). Management of Fluid Mud in Estuaries, Bays, and Lakes. II: Measurement, Modeling, and Management. Journal of Hydraulic Engineering, Vol. 133, n²1, pp 23-38. doi:10.1061/(ASCE)0733-9429(2007)133:1(23)

MEHTA A.J., HAYTER E.J., PARKER W.R., KRONE R.B., TEETER A.M. (1989). Cohesive sediments transport. Journal of Hydraulic Engineering, Vol. 115, $\mathrm{n}^{\circ}$ 8, pp 1076-1112. doi:10.1061/(ASCE)0733-9429(1989)115:8(1076)

ODD N.V.M., COOPER A.J. (1989). A Two-Dimensional Model of the Movement of Fluid Mud in a High Energy Turbid Estuary. Journal of Coastal Research, pp 185-193 PARCHURE T.M., MEHTA A.J. (1985). Erosion of soft cohesive sediment deposits. Journal of Hydraulic Engineering, Vol. 111(10), pp1308-1326. doi:10.1061/(ASCE)07339429(1985)111:10(1308)

PARTHENIADES E. (1965). Erosion and deposition of cohesive soils. Journal of Hydraulic Division, ASCE, Vol. 91(1), pp 105-137.

SANCHEZ M., LEVACHER D. (2008). Erosion of a mud from the Loire estuary by a flow. Bulletin of Engineering Geology and the Environment, Vol. 67, pp 597-605. doi:10.1007/s10064-008-0159-9

WINTERWERP J.C. (2002). On the flocculation and settling velocity of estuarine mud. Continental Shelf Research, Vol. 22, n9, pp 1339-1360. doi:10.1016/S0278-4343(02)00010-9 YIH C-S. (1967). Instability due to viscosity stratification. Journal of Fluid Mechanics, Vol. 27, n², pp 337-352. doi:10.1017/S0022112067000357 